\title{
Dermatomiositis y adenocarcinoma pancreático. Resolución tras gemcitabina
}

\section{Sr. Director:}

La dermatomiositis es una miopatía inflamatoria consistente en debilidad muscular principalmente proximal con o sin dolor y con alteraciones cutáneas asociadas. El rash cutáneo puede anteceder a los síntomas miopáticos más de un año. La primera asociación entre miositis y neoplasia fue publicada en 1916. Posteriores observaciones han sostenido el concepto de que los pacientes con polimiosistis y dermatomiositis tienen un incremento en el riesgo de padecer cáncer. Presentamos un caso de dermatomiositis asociada a adenocarcinoma pancreático con desaparición completa tras el tratamiento del tumor primario.

\section{Caso clínico}

Varón de 77 años de edad, sin antecedentes de interés. Consulta por enrojecimiento de la piel en tercio superior del tórax y parte proximal de ambos miembros junto con debilidad muscular proximal en brazos y piernas, eritema en heliotropo en párpados y pápulas violáceas en los nudillos de ambas manos, de un mes de evolución. Se realiza analítica destacando creatinkinasa 736 UI, LDH 545 UI, urea 63 mg/dl, CA 19.9 64,1 U/ml. Electromiograma: Hallazgos compatibles con proceso miopático inflamatorio. Biopsia muscular: Cambios inflamatorios compatibles con dermatomiositis. Con el diagnóstico de dermatomiositis se inicia tratamiento con prednisona $60 \mathrm{mg} /$ día y estudio: TAC toracoabdominal: Masa pancreática de $6 \mathrm{~cm}$ y lesiones hipodensas hepáticas. Punción-biopsia hepática: Adenocarcinoma. Se remite a Oncología. Tras 8 semanas de corticoides, sin mejoría de las lesiones cutáneas ni de la debilidad muscular, y ante el diagnóstico de adenocarcinoma pancreático en estadio IVB por metástasis hepáticas comenzamos tratamiento con gemcitabina $1250 \mathrm{mg} / \mathrm{m}^{2}$ días 1 y 8 cada 21 . Tras 2 ciclos de tratamiento se documenta resolución completa de las lesiones cutáneas, normalización de las enzimas musculares y recuperación de la fuerza. Actualmente el paciente se mantiene asintomático tras 6 ciclos de tratamiento y con respuesta menor del $50 \%$ de su enfermedad de base.

\section{Discusión}

La incidencia de dermatomiositis es de 1/100.000 personas. Se asocia a neoplasias en aproximadamente un $20 \%^{1-4}$ rango (6-60\%) por ello es aconsejable investigar la existencia de un tumor subyacente, aunque para algunos autores esta frecuencia ajustada a la edad no es muy diferente de la población general. Los cánceres asociados con más frecuencia son: ovario, pulmón, páncreas y estómago ${ }^{1}$, habiéndose descrito también en otros como en cabeza y cuello $^{2}$. La mayoría de las ocasiones, la neoplasia se diagnostica tras la dermatomiositis, habiéndose descrito neoplasias a los 5 años del diagnóstico. Estudios recientes indican que aunque la asociación se eleva en los mayores de 45 años, también es posible hallarla en enfermos jóvenes ${ }^{4}$.

Los síndromes paraneoplásicos son un conjunto de signos y síntomas originados por una neoplasia, que no se deben a invasión directa ni a metástasis de la misma. Pueden deberse a hormonas, factores de crecimiento u otras sustancias producidas por el, a interacciones antígeno-anticuerpo o a causas desconocidas. Excluyendo la anemia y la caquexia afectan a un $7-15 \%$ de los pacientes con cáncer ${ }^{3}$, aunque pudieran ser más frecuentes ${ }^{4}$. El origen de dermatomiositis como síndrome paraneoplásico no 
está claro, podría deberse a una alteración inmunitaria que originaría una infección o a una reacción inmunitaria cruzada entre tumor maligno, músculo y piel. El diagnóstico de dermatomiositis puede establecerse en un paciente con erupción cutánea, debilidad muscular, alteraciones electromiográficas y elevación de la creatinkinasa, sin necesidad de la biopsia muscular ${ }^{5}$. El principal tratamiento para la dermatomiositis son los corticoesteroides, tradicionalmente $0,5-1 \mathrm{mg} / \mathrm{kg}$ de prednisona al día. Un $25 \%$ de los pacientes no responde a este tratamiento, precisando otros agentes inmunosupresores como el metotrexato ${ }^{6}$ o la aziatropina, también se ha empleado con éxito las inmunoglobulinas intravenosas ${ }^{7}$. El tratamiento con corticoides debe mantenerse hasta la mejoría sintomática y la normalización de las enzimas musculares durante al menos un mes. Cuando se presenta como síndrome paraneoplásico el tratamiento es el del tumor primario, asociado a esteroides. Es de destacar en el caso que presentamos la falta de mejoría con esteroides a pesar de 8 semanas de tratamiento y la resolución completa tras el tratamiento del tumor primario.

\section{Vicente Baz ${ }^{1}$, C. Fernández Delgado ${ }^{2}$, R. Toda Savall ${ }^{2}$, A. Masabeu Urrutia ${ }^{2}$ ${ }^{1}$ Unidad de Oncología Médica ${ }^{2}$ Unidad de Medicina Interna Hospital de Palamós- Baix Empordá Palamós (Gerona)}

\section{Bibliografía}

1. Hill CL, Zhang Y, Sigurgeirsson Ba, Pukkala E, Mellemkjaer L, Airio A, et al. Frecuency of specific cancer types in dermatomyositis and polymyositis: a population-based study. Lancet 2001;357:96-100.

2. Mesia R, Sola C, Brunet J, Mendoza L, Sancho G, Lopez Pousa, et al. Dermatomyositis and head and neck cancer. Rev Clin Esp 1995;195:26-8.

3. Abeloff MD. Paraneoplastic síndromes. N Engl J Med 1987;317:1598-1600.

4. Albarrán-Hernández F, Cuadrado-Gómez LM, MonteroRuiz E, Martin Llorens MM. Síndromes Paraneoplásicos. Medicine (Ed. Española), 1995;6(79):3481-92.

5. Bohan A, Peter JB. Polymiositis and Dermatomiositis. N Engl Med 1975;292:344-347;403-407.

6. Villalba L, Adams EM. Update on therapy for refractory dermatomyositis and polymiositis. Curr Opin Rheumatol 1996;8:544-51.

7. Escriche D, de la Cruz J, Moreno MJ, Romero J. Efficacy of early treatment with intravenous immunoglobulins in severe forms of dermatomyositis. Med Clin 1995;21;104:79.

Correspondencia:

Dr. D. Vicente Baz

Unidad de Oncología Médica

Hospital Juan Ramón Jiménez

Avda. Ronda Norte, s/n

E-21006 Huelva

dvicentebaz@yahoo.es 